\title{
Controlled vocabulary in public health. An overview of the achievements to date
}

\author{
Barbara Niedźwiedzka • Katarzyna Czabanowska • \\ Rafal Śmietana
}

Received: 21 January 2008 / Accepted: 5 May 2008 / Published online: 25 June 2008

(C) Springer-Verlag 2008

\begin{abstract}
Aim The aim of the article is to present both published and unpublished information materials based on the literature review related to the accomplishments in the area of development and unification of the public health terminology. Background Public health is a multidisciplinary field that has long been struggling with the need to develop comprehensive and exhaustive terminology that would cover all related disciplines, facilitate accurate understanding of terms, aid information search and processing, as well as permit an accurate scientific communication among public health professionals. This is not an easy task given the theoretical and practical scope of the discipline.

Summary of the resources The following bibliographical databases were searched: LISA, PubMED, EMBASE, ERIC, as well as Internet websites devoted to public health issues, websites of the institutions operating in this area, and databases of projects financed by the European Union. All national and international reports in English, French, or German that dealt with the development of public health terminology in respective languages were included in the search. The glossaries, documents, databases, or web pages related to the activities of single institutions were excluded. Finally 13 glossaries were selected from among a number
\end{abstract}

B. Niedźwiedzka $(\bowtie)$

The Institute of Public Health,

Jagiellonian University Medical College,

Grzegórzecka 20, 31-531 Kraków, Poland

e-mail: mxniedzw@cyf-kr.edu.pl

K. Czabanowska

Institute of Public Health, JU Medical College,

Grzegórzecka 20, 31-531 Kraków, Poland

R. Śmietana

International House Kraków, Kraków, Poland of different dictionaries, glossaries, and thesauruses that cover the terms used in public health discipline or its subdisciplines. The selected glossaries were developed in order to unify the terminology with their intended circulation exceeding the narrow aim of collecting terminology for the purpose of one project or for cataloguing the resources of a particular institution.

Conclusion Until now, no one has developed one universal public health terminology, but various attempts related to its normalization are underway. The difficulties in communication, electronic collection, and transfer of information as well as differences in the understanding of public health terms have led to the process of unification. Such a process has been perceived not only in individual countries, but also internationally. There is hope that such works will finally result in the desired and internationally unified understanding of public health terms.

Keywords Public health · Vocabulary · Controlled · Dictionaries

\section{Background}

Public health as a multidisciplinary field on the one hand has special needs related to terminology development and on the other hand is confronted with major difficulties in its unification, unification that would greatly serve the processing of information and good communication among public health professionals. The task is not easy given how many disciplines contribute to the research related to the phenomena that have an impact on the health of populations. We are faced with a wide variety of activities and a multitude of data and data sources that stem from or crosscut: epidemiology, health policy, environmental re- 
search, health promotion and health education, health economics and management of organizations, psychology, and sociology, to name only some of the most important ones. Despite the difficulties, various attempts have been made to produce glossaries, thesauruses, or terminology lists that have tried to unify public health and health-related vocabulary.

The aim of the article is to present an overview, based on comprehensive literature and Internet sources review, related to the accomplishments in the area of development and unification of public health terminology. Included were vocabularies with a broad, national, or international scope of intended users. All dictionaries, thesauruses, and wordlists developed exclusively to cater for the needs of only a single institution, database, information center, or internet-based service have been disregarded.

\section{Summary of the resources}

A review of publications and available documents produced between the 1990s and June 2006 was conducted. Databases such as LISA, PubMED, EMBASE, and ERIC were searched using the following entries and keywords: PUBLIC HEALTH, vocabular*, dictionar*, thesaurus*, glossar*. Also Internet websites devoted to public health issues and websites of institutions and organizations operating in the field of interest were reviewed as well as databases of projects financed by the European Union (found in Cordis and on the Public Health Portal) related to the development of glossaries in the area of public health. We also collated all the reports and information related to the development of nationwide or international terminology lists in English, French, and German. No reports or documents were consulted that referred to glossaries that were designated for use by a single institution, database, or website.

Studies devoted to the development of a universal terminology in the field of public health are not that numerous. The few that were found demonstrate that the beginnings of the efforts geared towards the establishment of a universal terminology go back to the mid 1990s. It was then that both in Europe and the in United States professionals started to compile locally available glossaries for broader use. They included terms from multidisciplinary language glossaries, wordlists developed for specific purposes, and in order to explain the terms, current definitions or contexts were provided.

The first example is the Public Health Information Network (PHIN), created by the Centers for Disease Control and Prevention in the USA, which implemented Public Health Vocabulary usage and made available standard dictionaries that contained a number of specialist terms. PHIN vocabulary is supposed to enable the cooperation of information systems in various organizations operating in the domain of public health. This is one of the PHIN activities, whose overall goal is to provide a uniform architecture for the exchange of information needed by professionals and clients in the field of public health through compliance of the institutions to the terminology standards, data exchange protocols, codes used, etc., across the USA. One of such glossaries is the PHIN Controlled Health Thesaurus, CHT, a polyhierarchic thesaurus, which provides definitions of individual terms, their synonyms and interrelationships, as well as contains references to dictionaries, from which the terms originate. It is mainly based on the terms used in the Unified Medical Language System, created by the US National Library of Medicine. The system comprises over 100 area-specific dictionaries and coding systems, and contains over a million terms in medicine and related sciences (UMLS Metathesaurus. Fact Sheet 2004). However, since the review of individual dictionaries has demonstrated that none of them actually exhausts the useful terminology in the domain of public health, CHT also contains terms outside UMLS, which are related to, e.g., environmental health, occupational health, accidents, and bioterrorism (Almeida et al. 2003). PHIN vocabulary is available at: PHIN Vocabulary Access and Distribution System.

In 1994, the International Union for Health Promotion and Health Education, Regional Office for Europe (IUHPE/ EURO), one of whose aims is to facilitate information exchange among member organizations, started to work on a multilingual thesaurus in the area of health promotion and health education (Dorst 1998). This project was financed by the European Commission (DG V): the Program of Community Action for Health Promotion, Information, Education, and Training was implemented in cooperation with specialists in health terminology and health promotion, initially from four countries: the UK, France, Germany, and Holland. The thesaurus was based on the wordlist of the Netherlands Institute for Health Promotion and Disease Prevention (NGIZ), a thesaurus developed in Holland in 1982, and was used by the institutions that deal with health promotion and health education in this country. On completion of the first stage of work, the European Multilingual Thesaurus on Health Promotion comprised ca. 1,300 terms and allowed the use of databases in one of four languages in order to facilitate international cooperation and foster the dissemination and coordination of activities in the area of health promotion (Amis et al. 1996). The thesaurus contained the basic terms accepted at the international level and was developed in accordance with ISO recommendations on the creation of mono- and multilingual dictionaries, their form, arrangement, relationships, explanations, and references to other dictionaries [ISO 2788-1986(E); ISO 5964-1985 (E)]. The thesaurus 
was tested in several institutions in the UK, Scotland, and Holland, and in 1997 work was expanded to include Italy and Sweden. At the moment, the thesaurus is available in 12 languages: French, Danish, Dutch, English, Finnish, German, Italian, Portuguese, Swedish, Spanish, Norwegian, and Greek. The thesaurus is available at: http://www. hpmulti.net.

The year 2001 saw the emergence of a glossary whose scope was broader, exceeding the scope of health promotion and education-the Glossaire des Termes de Santé Publique Internationaux. This multilingual glossary is available in English, French, German, and Spanish, and is a product of a project financed by the European Commission, implemented by $15 \mathrm{EU}$ experts headed by Emmanuel Rusch. The glossary is made available by the Banque des Donnes Santé Publique (BDSP, France), and to a large extent, it is based on the Public Health Thesaurus (Thesaurus Santé Publique) developed by the Ecole Nationale de la Santé Publique (Rennes, France). The present, third review of the Thesaurus contains 6,186 terms, divided into 57 domains or the so-called microthesauruses, and 4,806 synonyms. The work on this thesaurus was started in the 1980s, with the key aim of the project being to develop a tool for indexing Public Health documents stored in the electronic Banque des Donnes Santé Publique (BDSP) and coordinated by the Ecole Nationale de la Santé Publique. The data collected by the BDSP came from a number of documenting institutions in France, hence the development of a uniform glossary of terms that describe documents maintained by individual institutions was crucial. The thesaurus is available at: http://www.bdsp.tm. $\mathrm{fr} / \mathrm{Glossaire/Default.asp.}$

Intensive work on controlled vocabulary in the area of public health is underway in the UK. The Health Development Agency [currently known as the National Institute for Health and Clinical Excellence (NICE)] developed the Public Health Information Thesaurus, based on the above-mentioned European Multilingual Thesaurus on Health Promotion. The thesaurus is used to index the publications listed in the Health Promotion Bibliographic Catalogue-HealthPromis, HDA's Evidence Base, as well as in the British portal Public Health Electronic Library. The HDA Public Health Information Thesaurus has been recently integrated with England's Public Health Observatories Public Health Information Tagging System (PHITS), and this way, a unified National Public Health Language (NPHL) was developed. The first version of this inventory of vocabulary was presented in December 2004. The National Public Health Language was created to permit uniform indexing and information search and to ensure efficient communication in the area of public health. This language, combining two important British terminologies, follows ISO recommendations and the APHO classification. It offers references to other glossaries operating in the public health subject area. NPHL comprises 1,571 terms in 10 thematic areas, as well as 340 synonyms. Work on the glossary is still underway and is geared towards its integration with the National Library's of Medicine MeSH (Medical Subject Headings), SNOMED (Systematized Nomenclature of Medicine), Government Category List and DoH Taxonomy. The NPHL is available at: http://www.nphl.nhs.uk or http://www.multites.com.

One should also mention glossaries that are narrowly specialized, yet very useful owing to the fact that their vocabulary is usually underrepresented in public health general dictionaries.

Among the multilingual thesauruses one needs to mention the General Multilingual Environmental Thesaurus, which was started in 1996 by the European Environment Agency (EEA) and its network-EIONET. GEMET was developed as a compilation of eight earlier multilingual glossaries and includes the basic environmental terminology. The 2001 revision is available in 17 European languages and has over 6,000 entries. The thesaurus is available through the European Environment Information and Observation Network website http://www.eionet.eu.int.

The National Information Center on Health Services Research and Health Care Technology (NICHSR) offers the "Terminology-A glossary of technical terms on the economics and finance of health services" http://www. nlm.nih.gov/nichsr/edu/healthecon/glossary.html.

The terminology related to the process of evaluating medical technologies-Health Care Technology AssessmentGlossary was developed by the National Information Center on Health Services Research and Health Care Technology (http://www.nlm.nih.gov/nichsr/ta101/ta10108.htm).

Many more or less extensive glossaries that may also be useful for Public Health professionals have been developed for the World Health Organization. Among them are the following:

- The Health Promotion Glossary developed by Don Nutbean of the WHO Collaboration Center for Public Health Promotion (University of Sydney, Australia) and published for the first time in 1986 is available at http://www.who.int/hpr/support.material.shtml

- The European Observatory on Health Systems and Policies Glossary can be found at http://www.euro. who.int/observatory/glossary/toppage

- The glossary of terms used in Health Impact Assessment is available at http://www.who.int/hia/ about/glos/en/index $2 . h t m l$

Numerous other WHO glossaries can be found using a search engine on the WHO website http://www.who.int/en/ by typing in the words "glossar," or "terminology," or 
"dictionar," each time adding a term that down-specifies a given area of interest.

- The most recent undertaking is: The Learners' Dictionary of Public Health for Students and Professionals. It has been developed in the framework of the European Leonardo da Vinci language competencies project by an international team of experts from Poland, Lithuania, the United Kingdom and Holland. The aim of the project was to develop a public health dictionary specifically targeted at the learners (the socalled learners' dictionary). Primary intended users of the glossary include students of public health, public health researchers and lecturers, teachers of specialist English, professionals and practitioners, who often work in international institutions, translators working for the EU and other international institutions, who should take into account the context of public health in their work. The definitions of terms, concepts, and expressions have been compiled in seven categories: epidemiology and biostatistics, environmental health and occupational health, health promotion, health policy, ethics and law, management, as well as economics and finance (Czabanowska 2008).

A word should also be said about terminology classifications that are also indispensable for the public health field and its professionals. They include, e.g., the International Classification of Diseases: 9th revision (ICD-9CM_2005), containing commonly adopted names of diseases and medical conditions, the International Statistical Classification of Diseases and Related Health Problems: 10th revision (ICD-10), as well as the International Classification of Primary Care (ICPC) for primary health care terminology, the WHO International Classification of Functioning, Disability and Health (http://www3.who.int/icf/onlinebrowser/icf.cfm). Likewise, the terminology used in the Universal Medical Device Nomenclature System that covers medical equipment, systems, and materials, developed by the American Institute ECRI and translated into a number of languages, can also be useful. (http://www.ecri.org/Products_and_Services/ Products/UMDNS/Default.aspx - UMDNS.)

\section{Conclusion}

To date, a single universal public health terminology has not been produced yet. Actually this does not seem to be possible given the present stage of development of the domain, in which no international consensus exists as to the actual organization of public health services; diversity of concepts and systems, which entails a comparable diversity of public health terms that describe this complex reality.
The same reasons for which the unification of public health terminology appears to be a necessity, such as difficulties in communication, misunderstandings arising from different meanings of the same terms, and overlapping of various terms across disciplines, constitute the most serious obstacles. Multidisciplinarity blurs conceptual borders of the domain, and varying public health responsibilities contribute to serious problems at the lexical level, such as the different understanding of the same terms or shifting semantic fields. These observations were confirmed in 2004 by a review of Public Health terminology across eight EU countries (France, Germany, Italy, Holland, Poland, Spain, Sweden, and the United Kingdom), which showed substantial diversity in the use of crucial terms (Kaiser and Mackenbach 2004).

The expansion of the Internet and institutional networks and intensive implementation of IT in health care institutions may intensify work on the unification and standardization of terminology. In order to be effective, public health activities have to be supported by complete and upto-date information supported by a uniform, comprehensive, and universal terminology. Normalized vocabulary, among others, is also important for the purposes of classification and education in the field of foreign language teaching or translation work. Hence, all the efforts to develop public health terminology undertaken not only at the national, but also at the international level, with significant contribution on the part of international organizations, especially the European Commission, cannot be underestimated. It is to be hoped that such efforts will bring about the unification of this terminology, similar to that achieved in medicine.

\section{Acknowledgements}

Source of support Leonardo da Vinci Language Competencies Project: PL/04/B/F/LA-174434 financed by the European Commission.

Conflict of interest statement The authors disclose any relevant associations that might pose a conflict of interest.

\section{References}

Almeida F, Bell M, Chavez E (2003) Controlled Health Thesaurus for the CDC Web redesign Project. AMIA Annu Symp Proc 2003:777

Amis R, Chevaillier G, Dorst L, Zimmermqan H (eds) (1996) Multilingual European Thesaurus on Health Promotion: DeutschEnglishFrancaisNetherlands. NIGZ, Woerden, 1996

Czabanowska K (2008) Learner's dictionary for students and professionals. Specialist English for Public Health. Jagiellonian University Publishers, Cracow

Dorst L (1998) Restoring the tower of Babel: building a multilingual thesaurus on health promotion. Health Libr Rev 15:33-39 
International Organization for Standardization (1985) Guidelines for the establishment and development of multilingual thesauri. ISO 5964-1985 (E)

International Organization for Standardization (1986) Guidelines for the establishment and development of monolingual thesauri. ISO 2788-1986(E)
Kaiser S, Mackenbach JP (2004) Public Health in Eight European Countries - An International Comparison of Terminology (unpublished article), July

Specialist English for European Public Health: Leonardo da Vinci Project no. PL/04/B/F/LA-174434 http://www.seeph.pro.mcg.pl/, and http://83.171.10.151/Zodynas/zodpakas/ 